\title{
Coupled cluster theory of strongly correlated spin- and electron-lattice systems: an illustration via a model exhibiting competition between magnetic order and dimerization
}

\author{
Raymond F Bishop $^{1}$ and Sven E Krüger ${ }^{2}$ \\ ${ }^{1}$ Department of Physics, University of Manchester Institute of Science \& Technology (UMIST), \\ PO Box 88, Manchester M60 1QD, UK \\ 2 Department of Electrical Engineering, IESK, Cognitive Systems, Universität Magdeburg, \\ PF 4120, 39016 Magdeburg, Germany
}

Received 31 January 2003

Published 20 August 2003

Online at stacks.iop.org/JPhysA/36/9143

\begin{abstract}
The coupled cluster method (CCM) of microscopic quantum many-body theory has become an ab initio method of first choice in quantum chemistry and many fields of nuclear, subnuclear and condensed matter physics, when results of high accuracy are required. In recent years it has begun to be applied with equal success to strongly correlated systems of electrons or quantum spins defined on a regular spatial lattice. One regularly finds that the CCM is able to describe accurately the various zero-temperature phases and the quantum phase transitions between them, even when frustration is present and other methods such as quantum Monte Carlo often fail. We illustrate the use and powerfulness of the method here by applying it to a square-lattice spin-half Heisenberg model where frustration is introduced by competing nearest neighbour bonds. The model exhibits the physically interesting phenomenon of competition between magnetic order and dimerization. Results obtained for the model with the CCM are compared with those found from spin-wave theory and from extrapolating the results of exact diagonalizations of small lattices. We show that the CCM is essentially unique among available methods in being able both to describe accurately all phases of this complex model and to provide accurate predictions of the various phase boundaries and the order of the corresponding transitions.
\end{abstract}

PACS numbers: 31.15.Dv, 05.30.Fk, 71.27.+a

\section{Introduction}

The coupled cluster method (CCM) [1] has become one of the most pervasive and most powerful of all ab initio formulations of quantum many-body theory. It has yielded numerical 
results which are among the most accurate available for a wide range of both finite and extended, physical and chemical systems defined on a spatial continuum [2]. This widespread success has spurred recent applications to similar quantum-mechanical systems defined on an extended regular spatial lattice.

Such lattice systems have become the subject of intense theoretical study. They include many examples of systems characterized by novel ground states which display quantum order in some region of the Hamiltonian parameter space, delimited by critical values which mark the position of the corresponding quantum phase transitions. The critical phenomena in these quantum-mechanical models often differ profoundly from those of their classical counterparts. Furthermore, the subtle correlations present usually cannot sensibly be treated by such standard many-body approaches as mean-field theory or perturbation theory. One of the key challenges for modern quantum many-body theory is to develop and exploit microscopic techniques capable of handling both these novel and the more traditional systems. Our recent work [3, 4] shows that the CCM is capable of bridging this divide.

In particular, we have shown how the systematic inclusion of multispin correlations for a wide variety of quantum spin-lattice problems can be very efficiently implemented with the CCM [3-12]. It is important to note that the method is not restricted to bipartite lattices or to non-frustrated systems, and can thus deal with problems where the quantum Monte Carlo (QMC) simulation techniques would be faced with the infamous 'minus sign problem'. The method is also easily extended to models of strongly interacting electrons on lattices, such as the Hubbard model [13-16], as well as to various Hamiltonian lattice field theories, such as the gauge theories U(1) [17] and SU(2) [18], and lattice chiral meson-field theory via the nonlinear O(4) sigma model [19].

In all of the above cases the CCM may readily be implemented to high orders (e.g., in a localized LSUB $n$ scheme) by the use of computer-algebraic techniques. Values for ground- and excited-state properties are thereby obtained which are fully competitive with those from other state-of-the-art methods, including the much more computationally intensive QMC techniques in cases where the latter can be applied. The raw LSUBn results themselves are generally excellent. They converge rapidly and can also provide valuable information on the quantum order and quantum criticality. In this paper, we illustrate these points by an application to a model with two types of nearest neighbour Heisenberg interactions [10, 12] on a two-dimensional square lattice. For certain values of the interaction strengths, the method exhibits the very interesting phenomenon of competition between magnetic order and dimerization.

\section{A typical model exhibiting competition}

We consider a spin-half Heisenberg model on a square lattice with two kinds of nearest neighbour bonds $J$ and $J^{\prime}$, as shown in figure 1 ,

$$
H=J \sum_{\langle i j\rangle_{1}} \mathbf{s}_{i} \cdot \mathbf{s}_{j}+J^{\prime} \sum_{\langle i j\rangle_{2}} \mathbf{s}_{i} \cdot \mathbf{s}_{j} .
$$

The expressions $\langle i j\rangle_{1}$ and $\langle i j\rangle_{2}$ indicate nearest neighbour bonds arranged in a regular zigzag pattern, as shown in figure 1 by the dotted and solid lines, respectively. Each square-lattice plaquette consists of three $J$ bonds and one $J^{\prime}$ bond. If $J^{\prime}$ and $J$ have different signs then the plaquettes are frustrated, whereas competition without frustration is realized for antiferromagnetic bonds $J^{\prime}>J>0$. A model with such a zigzag pattern of bonds has been treated previously by a variety of methods $[10,12,20,21]$. 

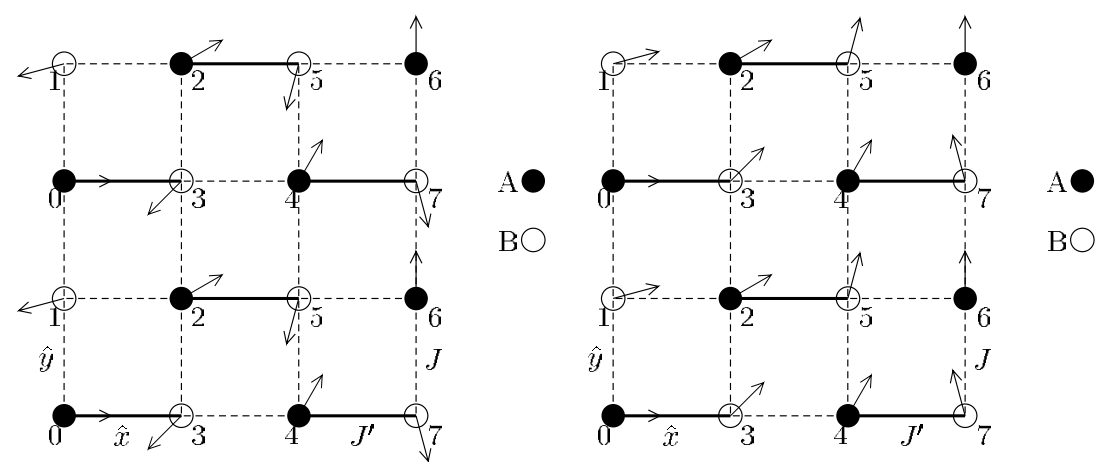

Figure 1. Illustration of the model ( $J$ bonds correspond to dotted lines and $J^{\prime}$ bonds to solid lines) and of the classical spiral state for antiferromagnetic $J=+1$ and ferromagnetic $J^{\prime}<-1 / 3$ (left graph), and vice versa $\left(J=-1, J^{\prime}>1 / 3\right)$ (right graph). As discussed in the text, both spiral states can be transformed into each other by reversing all of the spins on the $B$ sublattice.

The case of antiferromagnetic $J^{\prime}$ bonds with $J^{\prime}>J>0$ resembles the situation in bilayer systems and in the depleted square-lattice antiferromagnet $\mathrm{CaV}_{4} \mathrm{O}_{9}$, in which the competition between two different antiferromagnetic bonds leads to a phase transition from antiferromagnetic long-range order to quantum disorder with a finite gap. We see in this paper that the transition point obtained for the model of equation (1) is quite close to that obtained for the bilayer model [22].

For special values of $J, J^{\prime}$ the model represents (i) the spin-half Heisenberg antiferromagnet (ferromagnet) on the square lattice for $J=J^{\prime}=1\left(J=J^{\prime}=-1\right)$, (ii) the spin-half Heisenberg antiferromagnet (ferromagnet) on the honeycomb lattice for $J=1$, $J^{\prime}=0\left(J=-1, J^{\prime}=0\right)$ and (iii) the spin-1 Heisenberg antiferromagnet on the triangular lattice for $J=1, J^{\prime}=-\infty$.

\subsection{The classical ground state}

For $J=+1$ and $J^{\prime}>-1 / 3$ the Néel state is the classical ground state of the Hamiltonian of equation (1). At $J_{c}^{\prime}=-1 / 3$ there is classically a second-order phase transition to a ground state of helical nature (see figure 1), with a characteristic pitch angle $\Phi= \pm\left|\Phi_{\text {cl }}\right|$ given by

$$
\begin{array}{ll}
\left|\Phi_{\mathrm{cl}}\right|=0 & J^{\prime}>-\frac{1}{3} \\
\left|\Phi_{\mathrm{cl}}\right|=\arccos \left(\frac{1}{2} \sqrt{1-\frac{1}{J^{\prime}}}\right) & J^{\prime} \leqslant-\frac{1}{3}
\end{array}
$$

where the different signs correspond to the two chiralities [23] of this helical state. Note that for $\Phi=0$ this is just the Néel state. More generally, the pitch angle varies with $J^{\prime}$ from $\left|\Phi_{\mathrm{cl}}\right|=0$ for $J^{\prime}>-1 / 3$ to $\left|\Phi_{\mathrm{cl}}\right|=\pi / 3$ for $J^{\prime}=-\infty$. Note that $\left|\Phi_{\mathrm{cl}}\right|=\pi / 3$ (realized at $J^{\prime}=-\infty$ ) corresponds to the ground state of the spin-1 triangular lattice. For the $J=+1$ case we describe the directions of the spins $\mathbf{s}_{A}$ and $\mathbf{s}_{B}$, belonging to the $A$ and $B$ sublattices respectively, for the classical helical state with a characteristic angle $\Phi$ as follows [10] (and see figure 1),

$$
\begin{aligned}
& \mathbf{s}_{A}(\mathbf{R})=\hat{\mathbf{u}} \cos \mathbf{Q} \cdot \mathbf{R}+\hat{\mathbf{v}} \sin \mathbf{Q} \cdot \mathbf{R} \\
& \mathbf{s}_{B}(\mathbf{R}+\hat{x})=\hat{\mathbf{u}} \cos (\mathbf{Q} \cdot \mathbf{R}+\pi+3 \Phi)+\hat{\mathbf{v}} \sin (\mathbf{Q} \cdot \mathbf{R}+\pi+3 \Phi)
\end{aligned}
$$


where $\hat{\mathbf{u}}$ and $\hat{\mathbf{v}}$ are perpendicular unit vectors in the spin space, $\mathbf{R}$ runs over the sites of the sublattice $A$, and we have $\mathbf{Q}=(2 \Phi, 0)$ for the pitch vector $\mathbf{Q}$. We note that this general helical state does not have a periodicity in the $x$-direction because $\Phi$ is, in general, not of the form $m \pi / n$ with $m$ and $n$ integral. We also note that we have only three different angles between nearest neighbour spins, namely $\pm(\pi-\Phi)$ for the $J=1$ couplings and $(\pi-3 \Phi)$ for the coupling with $J^{\prime}$.

The maximum frustration for $J=+1$ is in the region around $J^{\prime} \approx-1$. Bearing in mind the situation for the $J_{1}-J_{2}$ model, one might expect that for the extreme quantum case (spin-half) quantum fluctuations might be able to open a window to a spin-liquid phase for a finite range of parameters around this region of maximum frustration. On the other hand, for strong antiferromagnetic bonds $\left(J^{\prime} \gg 1\right)$ there is, of course, no indication in the classical model for the breakdown of the Néel order.

By contrast to the quantum case, the classical model with $J=-1$ can be simply transformed into its counterpart with $J=+1$ considered above by the simultaneous substitutions $J \rightarrow-J, J^{\prime} \rightarrow-J^{\prime}, \mathbf{s}_{i \in B} \rightarrow-\mathbf{s}_{i \in B}$. Hence, the physics for the $J=-1$ sector of the classical model is essentially the same as for the $J=+1$ sector (see figure 1 ).

\section{The CCM for lattice Hamiltonians}

The starting point for any CCM calculation (see the overview in [1]) is the choice of a normalized model or reference state $|\Phi\rangle$, together with a set of mutually commuting multispin creation operators $C_{I}^{+}$which are defined over a complete set of many-body configurations $I$. The operators $C_{I}$ are the multispin destruction operators and are defined to be the Hermitian adjoints of the $C_{I}^{+}$. We choose $\left\{|\Phi\rangle ; C_{I}^{+}\right\}$in such a way that we have $\left\langle\Phi\left|C_{I}^{+}=0=C_{I}\right| \Phi\right\rangle$, $\forall I \neq 0$, with $C_{0}^{+} \equiv 1$.

For spin systems an appropriate choice for the CCM model state $|\Phi\rangle$ is often a classical spin state [3], in which the most general situation is that in which each spin can point in an arbitrary direction. After a local canonical transformation on each site to rotate each spin to the down $(-z)$ direction, we have

$$
|\Phi\rangle=|\cdots \downarrow \downarrow \downarrow \cdots\rangle \quad C_{I}^{+}=s_{r}^{+}, s_{r}^{+} s_{r^{\prime}}^{+}, s_{r}^{+} s_{r^{\prime}}^{+} s_{r^{\prime \prime}}^{+}, \ldots
$$

(where the indices $r, r^{\prime}, r^{\prime \prime}, \ldots$ denote any lattice site) respectively, for the model state and the multispin creation operators which consist of spin-raising operators only.

The CCM parameterizations of the ket and bra ground states are given by

$$
|\Psi\rangle=\mathrm{e}^{S}|\Phi\rangle \quad S=\sum_{I \neq 0} \mathcal{S}_{I} C_{I}^{+} \quad\langle\tilde{\Psi}|=\langle\Phi| \tilde{S} \mathrm{e}^{-S} \quad \tilde{S}=1+\sum_{I \neq 0} \tilde{\mathcal{S}}_{I} C_{I} .
$$

To find the ket-state and bra-state correlation coefficients we require that the expectation value $\bar{H}=\langle\tilde{\Psi}|H| \Psi\rangle$ be stationary with respect to all variations in the bra-state and ket-state correlation coefficients. Thus, the CCM ket-state equations, for example, are given by

$$
\left\langle\Phi\left|C_{I} \mathrm{e}^{-S} H \mathrm{e}^{S}\right| \Phi\right\rangle=0 \quad \forall I \neq 0 .
$$

The problem of determining the CCM equations now becomes a pattern-matching exercise of the $\left\{C_{I}\right\}$ to the terms in $\mathrm{e}^{-S} H \mathrm{e}^{S}$ in equation (6). The excited states may also be parameterized in an analogous fashion (see [1-4] for details).

We now use the lattice symmetries in order to find all different possible configurations with respect to the point and space group symmetries (of both the lattice and Hamiltonian) with up to $n$ spin flips spanning a range of no more than $n$ adjacent lattice sites (LSUB $n$ approximation), and these are referred to as the fundamental configurations or clusters. Details of the CCM 
Table 1. Number of fundamental ground-state configurations of the LSUB $n$ approximation for the Hamiltonian of equation (1), using a Néel state $(\Phi=0)$ and a helical state $(\Phi \neq 0)$ for the CCM model state, and the number of fundamental excited-state configurations using the Néel model state only, for $n=2,4,6,8$.

\begin{tabular}{lrrr}
\hline & \multicolumn{2}{c}{ Ground state } & Excited state \\
\cline { 2 - 3 } LSUB $n$ & $\Phi=0$ & $\Phi \neq 0$ & $\Phi=0$ \\
\hline 2 & 3 & 5 & 1 \\
4 & 22 & 76 & 16 \\
6 & 267 & 1638 & 331 \\
8 & 4986 & 42160 & 7863 \\
\hline
\end{tabular}

computational algorithm for spin quantum number $s=1 / 2$ are given in $[3,9]$ and for general $s$ in [4]. The resulting numbers of CCM LSUB $n$ configurations for the present model for $n \leqslant 8$ are given in table 1 , using both the Néel and the spiral states as model states.

\section{Results}

\section{1. $J=+1 ; J^{\prime}>0$ : competition without frustration (Néel versus dimer)}

Let us consider first the regime where $J=+1, J^{\prime}>0$. The two terms in the Hamiltonian are not 'frustrating' each other, but they do compete since the first $(J)$ term favours Néel long-range order on the honeycomb lattice on which it acts in the absence of the other term $\left(J^{\prime}=0\right)$, while the second $\left(J^{\prime}\right)$ term by itself favours an uncorrelated product state of local pair singlets (as discussed in more detail below). We recall that no such competition exists in this regime in the classical model.

Mean field approach. We start with a simple mean-field (MF) like description of the orderdisorder transition. The corresponding uncorrelated MF state for Néel long-range order (LRO) is the Néel state $\left|\phi_{\mathrm{MF}_{1}}\right\rangle=|\uparrow \downarrow \uparrow \cdots\rangle$, and for the dimerized singlet state it is the rotationally invariant product state of local pair singlets $\left|\phi_{\mathrm{MF}_{2}}\right\rangle=\prod_{i \in A}\left[\left|\uparrow_{i} \downarrow_{i+\hat{x}}\right\rangle-\left|\downarrow_{i} \uparrow_{i+\hat{x}}\right\rangle\right] / \sqrt{2}$ where $i$ and $i+\hat{x}$ correspond to those sites which cover the $J^{\prime}$ bonds. In order to describe the transition between both states, we consider an uncorrelated product state interpolating between $\left|\phi_{\mathrm{MF}_{1}}\right\rangle$ and $\left|\phi_{\mathrm{MF}_{2}}\right\rangle$ of the form $[10,24]$

$$
\left|\Psi_{\mathrm{MF}}(t)\right\rangle=\prod_{i \in A} \frac{1}{\sqrt{1+t^{2}}}\left[\left|\uparrow_{i} \downarrow_{i+\hat{x}}\right\rangle-t\left|\downarrow_{i} \uparrow_{i+\hat{x}}\right\rangle\right]
$$

We have $\left|\Psi_{\mathrm{MF}}(t=0)\right\rangle=\left|\phi_{\mathrm{MF}_{1}}\right\rangle$; and $\left|\Psi_{\mathrm{MF}}(t=1)\right\rangle=\left|\phi_{\mathrm{MF}_{2}}\right\rangle$. We minimize $E=$ $\left\langle\Psi_{\mathrm{MF}}|H| \Psi_{\mathrm{MF}}\right\rangle$ with respect to $t$ and obtain

$$
\begin{array}{ll}
E_{\mathrm{MF}} / N=-\frac{3 J^{\prime}}{8}-\frac{1}{24 J}\left(3 J-J^{\prime}\right)^{2} & J^{\prime} \leqslant 3 J \\
E_{\mathrm{MF}} / N=-\frac{3 J^{\prime}}{8} & J^{\prime}>3 J
\end{array}
$$

for the energy per site. For the sublattice magnetization $m \equiv\left\langle\Psi_{\mathrm{MF}}\left|s_{i \in A}^{z}\right| \Psi_{\mathrm{MF}}\right\rangle$ we get $m=\sqrt{\left(3 J-J^{\prime}\right)\left(3 J+J^{\prime}\right)} /(6 J)$ for $J^{\prime} \leqslant 3 J$ and $m=0$ otherwise. Note that $m$ vanishes at a critical point $J_{c}^{\prime}=3 J$, and that the critical index is the MF index $1 / 2$. Equation (8) may be rewritten in terms of $m$ as $E_{\mathrm{MF}} / N=-\frac{1}{8} J^{\prime}-\frac{1}{4} J^{\prime} \sqrt{1-4 m^{2}}-\frac{3}{2} J m^{2}$, and figure 2 illustrates that the dependence of $E_{\mathrm{MF}}$ on $m$ corresponds to the typical scenario of a secondorder transition. We can expand $E_{\mathrm{MF}}$ up to the fourth order in $m$ near the critical point and 

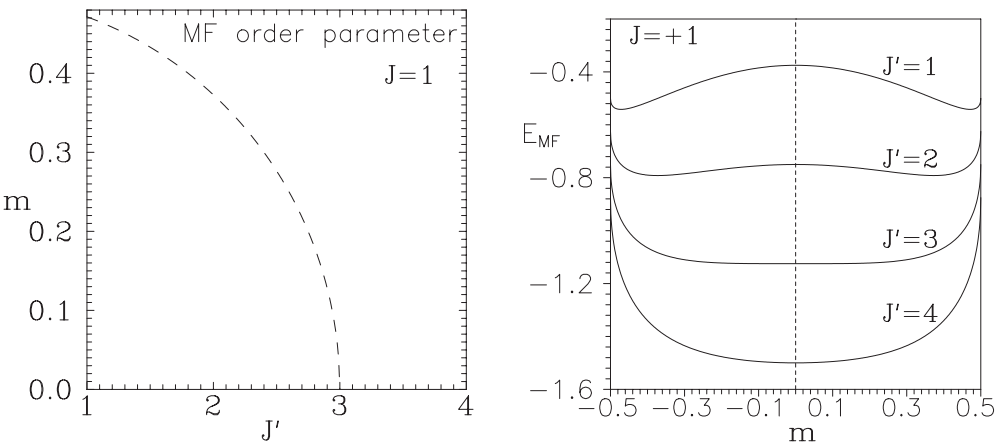

Figure 2. Sublattice magnetization versus $J^{\prime}$ (for $J=1$ ) (left graph), and the corresponding energy versus sublattice magnetization (right graph), using the mean-field approach of equation (7).
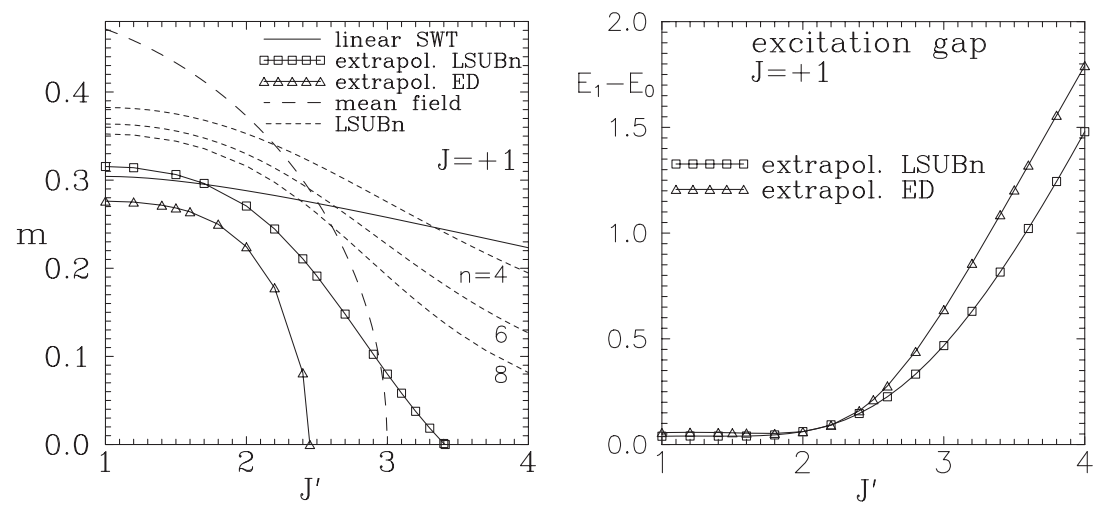

Figure 3. Sublattice magnetization (left graph) and excitation gap (right graph) versus $J^{\prime}$, for the case $J=+1$

find a Landau-type expression, given by $E_{\mathrm{MF}} / N=-\frac{3}{8} J^{\prime}+\frac{1}{2}\left(J^{\prime}-3 J\right) m^{2}+\frac{1}{2} J^{\prime} m^{4}$. However, as discussed elsewhere for a similar magnetic model for $\mathrm{CaV}_{4} \mathrm{O}_{9}$ [25], MF theory probably does not describe the critical behaviour correctly.

$C C M$. Let us therefore now apply a high-order CCM approach (for details see [3, 10]) to this model. We set the classical collinear Néel state to be the reference state $|\Phi\rangle$. We calculate the ground-state (GS) wavefunction, $|\Psi\rangle=\mathrm{e}^{S}|\Phi\rangle$, within the LSUB $n$ approximation scheme up to $n=8$, and extrapolate to $n \rightarrow \infty$. The CCM results for the order parameter are shown in the left graph of figure 3 and they are compared to results of linear spin-wave theory (SWT), exact diagonalization (ED) of $N=16,18,20,26,32$ sites and the MF theory. The CCM is able to describe correctly the order-disorder transition, whereas conventional SWT cannot (for more details concerning the SWT and ED results see [10]). The critical value predicted by extrapolation of the LSUB $n$ results is, however, found to be slightly too large. We may also consider the inflection points of $m$ versus $J^{\prime}$ for the LSUB $n$ approximations. It is assumed that the true $m\left(J^{\prime}\right)$-curve will have a negative curvature up to the critical point. Thus we might expect that (for increasing $n$ ) the inflection point approaches the critical point. We find the corresponding inflection points at $J^{\prime}=3.1(n=2), J^{\prime}=3.0(n=4), J^{\prime}=2.9(n=6)$ and $J^{\prime}=2.85(n=8)$, indicating a critical value $J_{c}^{\prime}$ somewhere between $2.5 J$ and $3 J$. Note that the estimation of $2.5 \lesssim J_{c}^{\prime} / J \lesssim 3$ is consistent with results of series expansions and exact 

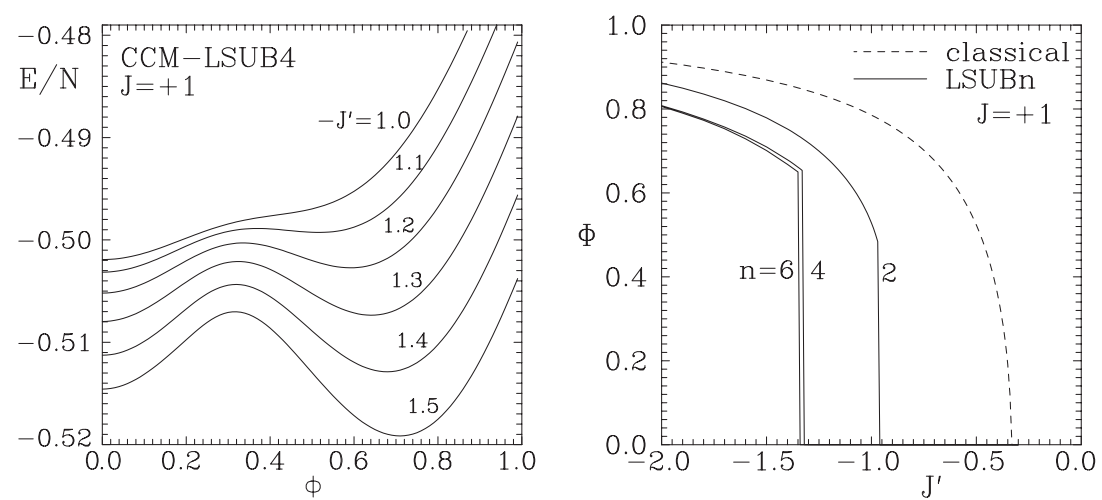

Figure 4. Energy versus quantum pitch angle for LSUB4 (left graph) and quantum pitch angle versus $J^{\prime}$ (right graph) for the case $J=+1$. Note that $\Phi=0$ corresponds to the Néel state.

diagonalizations $[10,20]$. The breakdown of Néel long-range order (LRO) due to singlet formation is also accompanied by the opening of an excitation gap between the singlet GS and the first triplet excitation. This behaviour is well described by the CCM (right graph of figure 3) which predicts that the gap opens in the range $2 J<J^{\prime}<3 J$ (and note that the non-zero gap below $2 J$ is a result of the limited accuracy of the extrapolation).

\section{2. $J=+1 ; J^{\prime}<0$ : competition with frustration (Néel versus spiral)}

In this and the following, subsection, we consider the frustrated regimes of the model where $J$ and $J^{\prime}$ have opposite signs. We first consider the case $J=+1, J^{\prime}<0$ where the Néel and spiral phases compete, and in section 4.3 consider the case $J=-1, J^{\prime}>0$ where the ferromagnetic and spiral phases compete. In both cases the ED technique for finite-size lattices is expected to be less useful because of the incommensurate nature of the classical spiral states. By contrast, the CCM considers the limit $N \rightarrow \infty$ from the outset and should have no such problem dealing with incommensurate states. In both cases we use the corresponding classical state of section 3.1 as the CCM model state. However, since quantum fluctuations can clearly change the spiral pitch angle from its classical value, we determine the 'quantum pitch angle' $\Phi$ by minimizing $E_{\mathrm{LSUB} n}(\Phi)$ with respect to $\Phi$ in each order $n$.

Results for $E(\Phi)$ and $\Phi\left(J^{\prime}\right)$ are shown in figure 4 for the case $J=+1, J^{\prime}<0$. A number of key points emerge. Firstly, it is clear that the CCM continues to yield a consistent description of the system in both these collinear and spiral phases. Secondly, the quantum Néel state remains the ground state up to much stronger frustration than in the classical case. This is in keeping with the general observation that quantum fluctuations in spin systems tend to favour collinear spin structures over non-collinear ones. Thirdly, and very strikingly, we observe that in this case the quantum fluctuations also change the order of the phase transition from second order in the classical case to first order in the quantum case.

\section{3. $J=-1 ; J^{\prime}>0$ : competition with frustration (ferro versus spiral)}

We now contrast the case considered in section 4.2 with the case where $J=-1, J^{\prime}>0$. Whereas in the classical model we again have a second-order transition between collinear and spiral phases, the quantum model in these two regimes behaves quite differently. Although the collinear antiferromagnetic phase contains strong quantum fluctuations as we saw above, the fully polarized, collinear ferromagnet contains no such fluctuations. The corresponding 

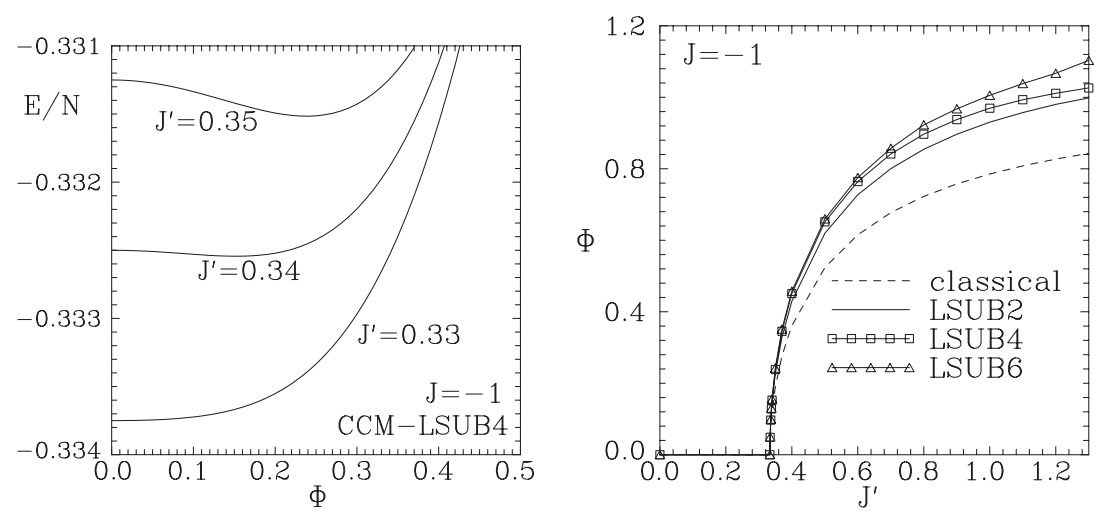

Figure 5. Energy versus quantum pitch angle for LSUB4 (left graph) and quantum pitch angle versus $J^{\prime}$ (right graph) for the case $J=-1$. Note that $\Phi=0$ corresponds to the fully polarized ferromagnetic state.

results for $E(\Phi)$ and $\Phi\left(J^{\prime}\right)$ are displayed in figure 5. In this case the order of the transition from the collinear (ferromagnetic) to the non-collinear (spiral) phase remains second order, as in the classical case. Furthermore, the quantum critical point remains exactly at the classical value, $J^{\prime} / J=-1 / 3$.

\subsection{Comparison of the various cases}

The difference between the two cases considered in sections 4.2 and 4.3 is particularly illustrated by figure 6 which contrasts the behaviour of the order parameter, namely the on-site magnetic moment $m$, in the two regimes. For $J=+1$ there exists a discontinuity in $m$ at every level of LSUB $n$ approximation. This is a reflection of the behaviour observed in $E(\Phi)$ in figure 4, where a local minimum at $\Phi \neq 0$ appears for $J^{\prime} \lesssim-1.1$, which for values $J^{\prime} \lesssim-1.35$ becomes a global minimum. The extrapolation of $m$ to the exact $n \rightarrow \infty$ limit does, however, become imprecise close to the phase transition point, and we cannot decide with certainty whether the order parameter does vanish at the transition point or, indeed, over a small finite regime around it. Figure 6 displays a minimum extrapolated value of $m \approx 0.05$ at $J_{c}^{\prime} \approx-1.2$. We cannot exclude entirely a disordered quantum spin-liquid phase with $m=0$ caused by quantum fluctuations plus frustration, but if it does exist it can clearly occur only over a very narrow regime in $J^{\prime}$ around $-1.5 \lesssim J^{\prime} \lesssim-1.1$. The most likely scenario however is a single phase boundary at a value $J_{c}^{\prime} \approx-1.35$ between spiral and Néel phases, with no intervening separate spin-liquid phase.

By contrast, figure 6 shows a smooth change in $m$ at the critical point $J_{c}^{\prime}=1 / 3$ for $J=-1$. For increasing $J^{\prime}>J_{c}^{\prime}$ the spiral magnetic order becomes weaker and finally vanishes at $J^{\prime} \approx 1$. The underlying reason for this is again local singlet formation (i.e., dimerization), as discussed in section 4.1. On the other hand, a much smaller critical strength $J_{c}^{\prime}$ is needed for dimerization than in the case $J=+1$ (where $2.5 \lesssim J_{c}^{\prime} \lesssim 3$ ), due to the effects of frustration which now assist in the formation of local singlets [24].

Finally, in figure 7 we show both the ground-state energy $E\left(J^{\prime}\right)$ and the order parameter $m\left(J^{\prime}\right)$ in the case $J=+1$ over the regimes spanning the spiral, Néel and dimer-like phases. We observe excellent agreement between the CCM results and the results from exact diagonalization (ED) for $J^{\prime}>0$. By contrast, spin-wave theory (SWT) calculations [21] show a significant deviation for larger $J^{\prime} \gg 1$. These latter are obviously poor since they 

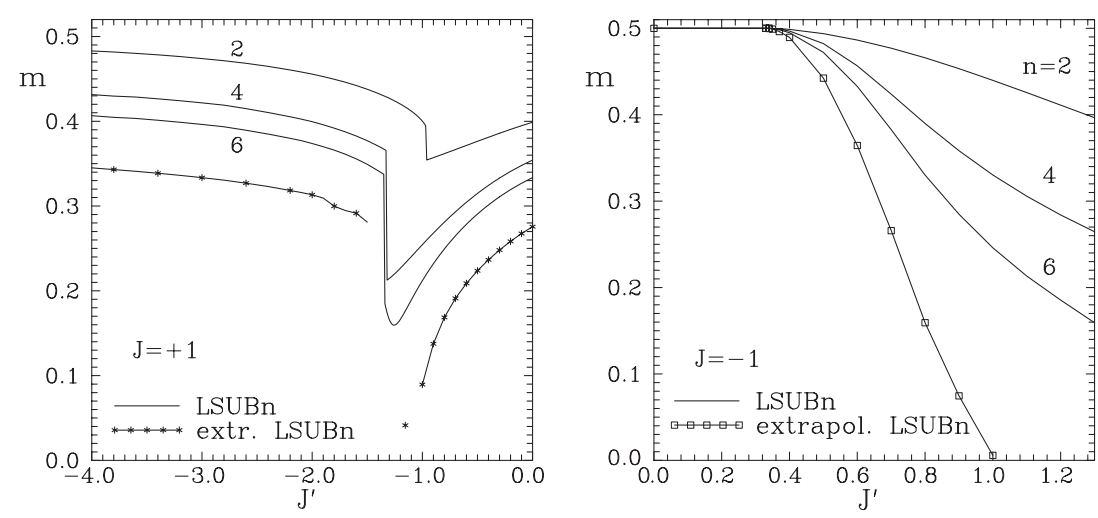

Figure 6. On-site magnetic moment versus $J^{\prime}$ for $J=+1$ (left graph) and $J=-1$ (right graph).
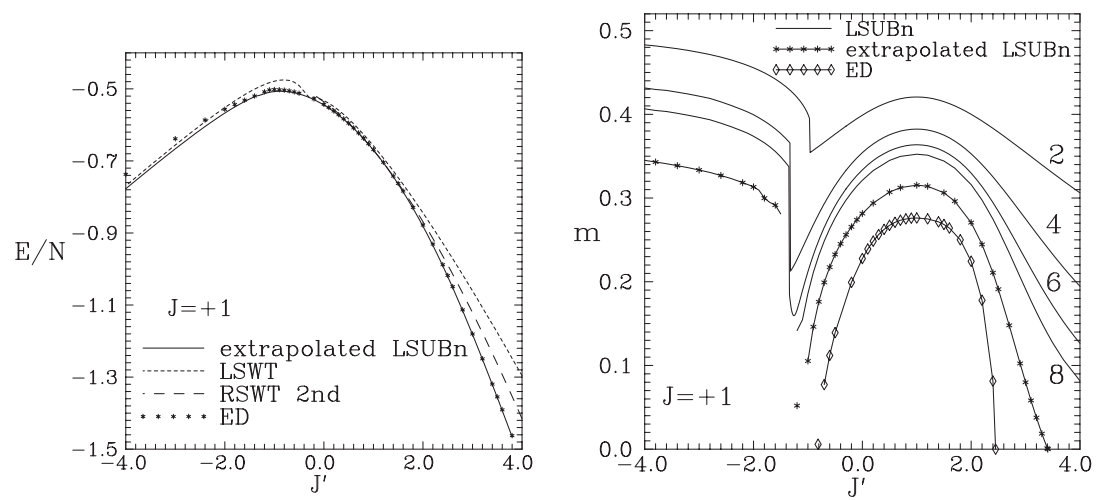

Figure 7. Left: ground-state energy versus $J^{\prime}$, for the case $J=+1$, for the extrapolated CCM LSUB $n$ approximations, in comparison with results of spin-wave theory (SWT) (linear and secondorder renormalized) [21], and with the extrapolated results of exact diagonalization (ED) data. Right: ground-state magnetic order parameter versus $J^{\prime}$, for the case $J=+1$, for the CCM LSUB $n$ approximation. The results are compared (for the Néel region only) with the extrapolated results of exact diagonalization (ED) data. Note that both extrapolated results fit poorly in a region around $J^{\prime} \approx-1$, and we therefore plot them here as isolated points, omitting the solid lines.

lie above the simple variational upper bound of equation (8). The CCM and ED results, by comparison, lie slightly below the mean-field variational result.

We note that although the Néel state is the starting reference state for both CCM and SWT calculations, the CCM is much better able than SWT to describe the transition to the rotationally invariant disordered state and hence to its limiting form, namely the fully dimerized state of equation (7) with $t=1$. For example, even the simplest LSUB2 CCM approximation gives the correct asymptotic behaviour for the ground-state energy, $E / N \rightarrow-3 J^{\prime} / 8$ as $J^{\prime} \rightarrow \infty$, whereas SWT does not.

\section{Discussion and conclusions}

In this paper, we have investigated the zero-temperature phase transitions of a spin-half Heisenberg system on the square lattice in which frustration has been introduced via competing nearest neighbour bonds. The main results of our treatment are as follows: 
- Quantum fluctuations plus competition without frustration are able to destroy Néel longrange order by local singlet formation. This is a pure quantum effect and has no classical counterpart. The control parameter is the strength of the competition and the breakdown of Néel ordering is accompanied by the opening of a spin gap. Standard SWT (even to higher orders) fails to describe this transition, whereas the CCM describes both the order parameter and the gap satisfactorily. Since we have no frustration, most standard techniques (e.g., QMC) are applicable and a quantitative description is possible. As was discussed in [25], the critical properties seem to correspond to the 3D classical Heisenberg model.

- Competition due to frustration was found to give more complex magnetic properties. In the model considered we have a second-order transition between collinear (antiferromagnetic or ferromagnetic) and non-collinear (spiral) states driven by frustration in the classical case. In the quantum spin-half model, standard techniques (e.g., QMC) are not applicable due to the violation of Marshall's sign rule. By contrast, the CCM provides a consistent description of collinear, non-collinear, and disordered phases. Furthermore, we find a strong influence of quantum fluctuations on the nature of the collinear-non-collinear transition, and quantum fluctuations (which favour collinear ordering) may change the second-order classical transition to a first-order quantum transition. If quantum fluctuations are suppressed in the collinear phase, the transition to the spiral phase is similar for the quantum and classical models.

It is our belief that the excellent results obtained, both for the present model and a number of other spin-lattice systems treated previously, are sufficiently encouraging to contemplate now applying the CCM to a wide range of strongly correlated magnetic lattice systems that alternative techniques might have difficulty in treating. An obvious example that we intend to investigate is the physically similar, but technically more demanding, system of the depleted square-lattice antiferromagnet $\mathrm{CaV}_{4} \mathrm{O}_{9}$.

Based on the success of the CCM to describe the model considered here and other complex magnetic lattice models, it is also of interest to enquire about other possible extensions. One particularly important such extension would be to non-zero temperatures, in order to investigate quantitatively, and in a fully $a b$ initio microscopic framework, the competing effects of quantum and thermal fluctuations on the full phase diagram of a given model system. One of the present authors (RFB) is actively considering such an extension.

\section{References}

[1] Bishop R F 1998 Microscopic Quantum Many-Body Theories and Their Applications (Lecture Notes in Physics vol 510) ed J Navarro and A Polls (Berlin: Springer) p 1

[2] Bishop R F 1991 Theor. Chim. Acta 8095 Bartlett R J 1989 J. Phys. Chem. 931697

[3] Zeng C, Farnell D J J and Bishop R F 1998 J. Stat. Phys. 90327

[4] Farnell D J J, Bishop R F and Gernoth K A 2002 J. Stat. Phys. 108401

[5] Bishop R F, Hale R G and Xian Y 1994 Phys. Rev. Lett. 733157

[6] Farnell D J J, Krüger S E and Parkinson J B 1997 J. Phys.: Condens. Matter 97601

[7] Bishop R F, Farnell D J J and Parkinson J B 1998 Phys. Rev. B 586394

[8] Bishop R F, Farnell D J J and Zeng C 1999 Phys. Rev. B 591000

[9] Bishop R F, Farnell D J J, Krüger S E, Parkinson J B, Richter J and Zeng C 2000 J. Phys.: Condens. Matter 126887

[10] Krüger S E, Richter J, Schulenburg J, Farnell D J J and Bishop R F 2000 Phys. Rev. B 6114607

[11] Farnell D J J, Bishop R F and Gernoth K A 2001 Phys. Rev. B 63 220402(R)

[12] Krüger S E and Richter J 2001 Phys. Rev. B 64024433

[13] Roger M and Hetherington J B 1990 Europhys. Lett. 11255 
[14] Lo C F, Manousakis E and Wang Y L 1991 Phys. Lett. A 15642

[15] Petit F and Roger M 1994 Phys. Rev. B 493453

[16] Bishop R F, Xian Y and Zeng C 1995 Int. J. Quantum Chem. 55181

[17] Baker S J, Bishop R F and Davidson N J 1996 Phys. Rev. D 532610

[18] Llewellyn Smith C H and Watson N J 1993 Phys. Lett. B 302463 Schütte D, Zheng W H and Hamer C J 1997 Phys. Rev. D 552974

Wichmann A, Schütte D, Metsch B C and Wethkamp V 2002 Phys. Rev. D 65094511

[19] Ligterink N E, Walet N R and Bishop R F 1998 Ann. Phys., NY 26797

Ligterink N E, Walet N R and Bishop R F 2001 Phys. Rev. E 63037103

[20] Singh R R P, Gelfand M P and Huse D A 1998 Phys. Rev. Lett. 612484

[21] Ivanov N B, Krüger S E and Richter J 1996 Phys. Rev. B 532633

[22] Sandvik A W and Scalapino D J 1994 Phys. Rev. Lett. 722777

[23] Villain J 1977 J. Phys. C: Solid State Phys. 104793

[24] Gros C, Wenzel W and Richter J 1995 Europhys. Lett. 32747

[25] Troyer M, Imada M and Ueda K 1997 J. Phys. Soc. Japan 662957 